\title{
K. Barlai
}

Konkoly Observatory, Budapest

The O-C curves - widely used for investigating the period behaviour of variable stars - are generally constructed under the assumption that the period changes are linear in time. If it is indeed so, the main trend of the diagram should outline a positive or negative parabola and $\beta$ values are determined to characterize the curvature of the parabolae, i.e. the rate of change of the period. This rate, however, even in the case of $\omega$ Cen does not indicate exact parabola (Belserene, 1973).

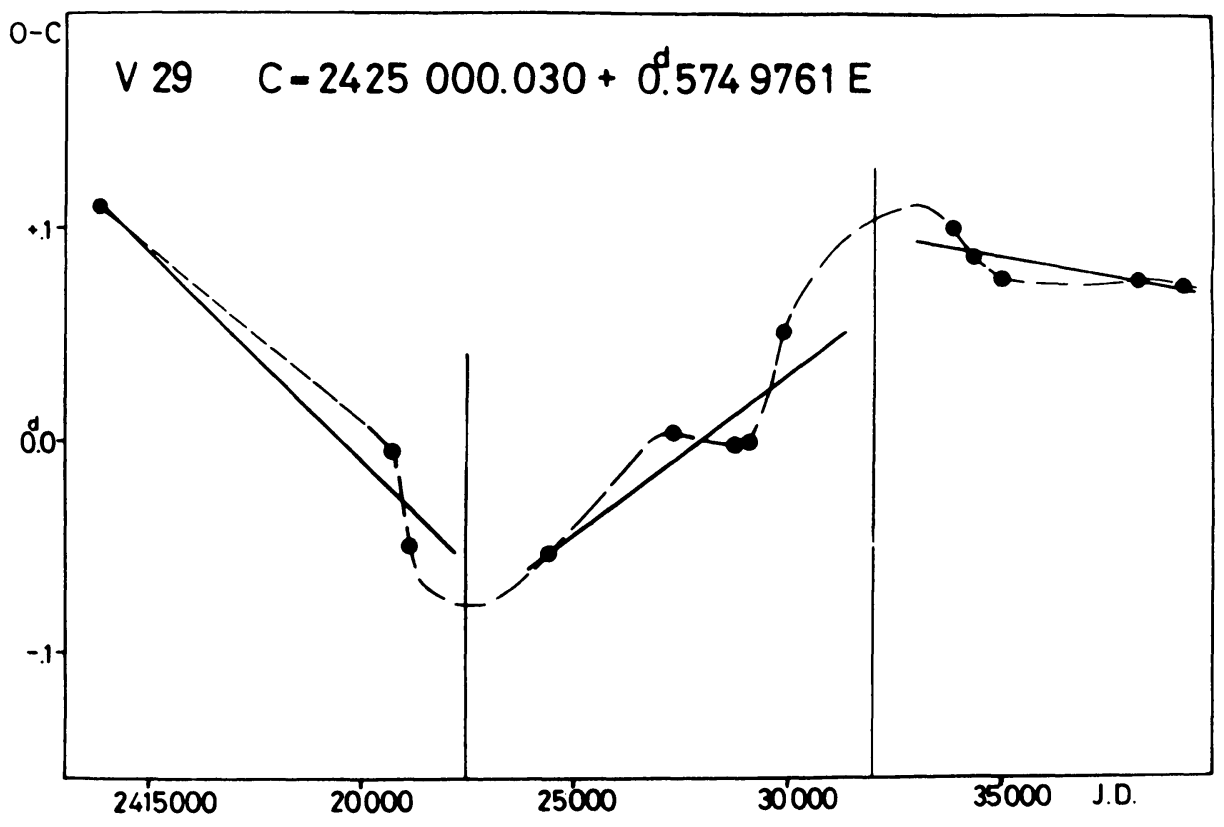

Fig. 1. An example for determination of the average periods. 457

A. Maeder and A. Renzini (eds.), Observational Tests of the Stellar Evolution Theory, 457-460.

(C) 1984 by the IAU. 
The various shapes of the O-C diagrams obtained for 49 RR Lyrae variables in the globular cluster M 15 convincingly suggest (Barlai, 1984) that the concept of a linear period change does not work in our case. Only a minor part of the O-C residuals could be approximated by parabolae or straight lines (Szeidl, 1975). In view of this, when investigating the trend of the period changes in M 15 a method was worked out which would need no specific preliminary assumptions concerning the character of the period changes.

The time interval covered by the observations (J.D.2413700 - J.D. 2440000) has been divided into three parts. Within these time intervals three average periods have been determined for each RR Lyrae variable from the points of their $0-C$ residuals. By subtracting the average periods belonging to the subsequent intervals two series of $\Delta \mathrm{P}$ values have been obtained. For each star the single $\Delta P$ values have been related to the time elapsing between the corresponding average epochs, i.e. the average abscissa values belonging to the straight line representing the average periods valid within the subsequent sections of time. (See Fig. 1 as an example.)

Having performed this calculation for all the RR Lyrae variables measurable on our plates in $\mathrm{M} 15$, two series of $\Delta \mathrm{P} / \Delta \mathrm{t}$ values have been obtained. The members of one single series practically all belong to the same section of time. We have concerned ourselves here with the distribution of these $\Delta \mathrm{P} / \Delta \mathrm{t}$ values.

Both of the resulting histograms (Fig.2) have a prominent peak at zero, are roughly symmetrically situated around the zero point, and this symmetrical part contains the majority of the data. These features and the fact that the observed period changes can be explained by different random processes going on inside an RR Lyrae star (Sweigart and Renzini, 1979) do not contradict a normal distribution. So a Gaussian fitting has been attempted although the data available are not very numerous from a statistical point of view.

Having fitted a Gaussian curve upon the central part of the distribution it became obvious that the broad wings cannot be included in the curve. Single stars are to be found even at a distance as far as $\sim 150$ ( $V$ 15). A considerable part of the variables studied (11) belongs to the wings and these stars underwent strong and rapid period changes during the seven decades of the observations. In several cases a jump from the left wing to the right can be observed and vice versa. For example variables 2 and 13 appear in the left wing of distribution $I$ and are to be found on the right wing of distribution II.

The stars belonging to the Gaussian distribution exhibit "noisy" period behaviour. The evolutionary trend does not emerge above this noise level.

The method outlined here has enabled us to take into account all kinds of period changes without being prejudiced against the shape of 


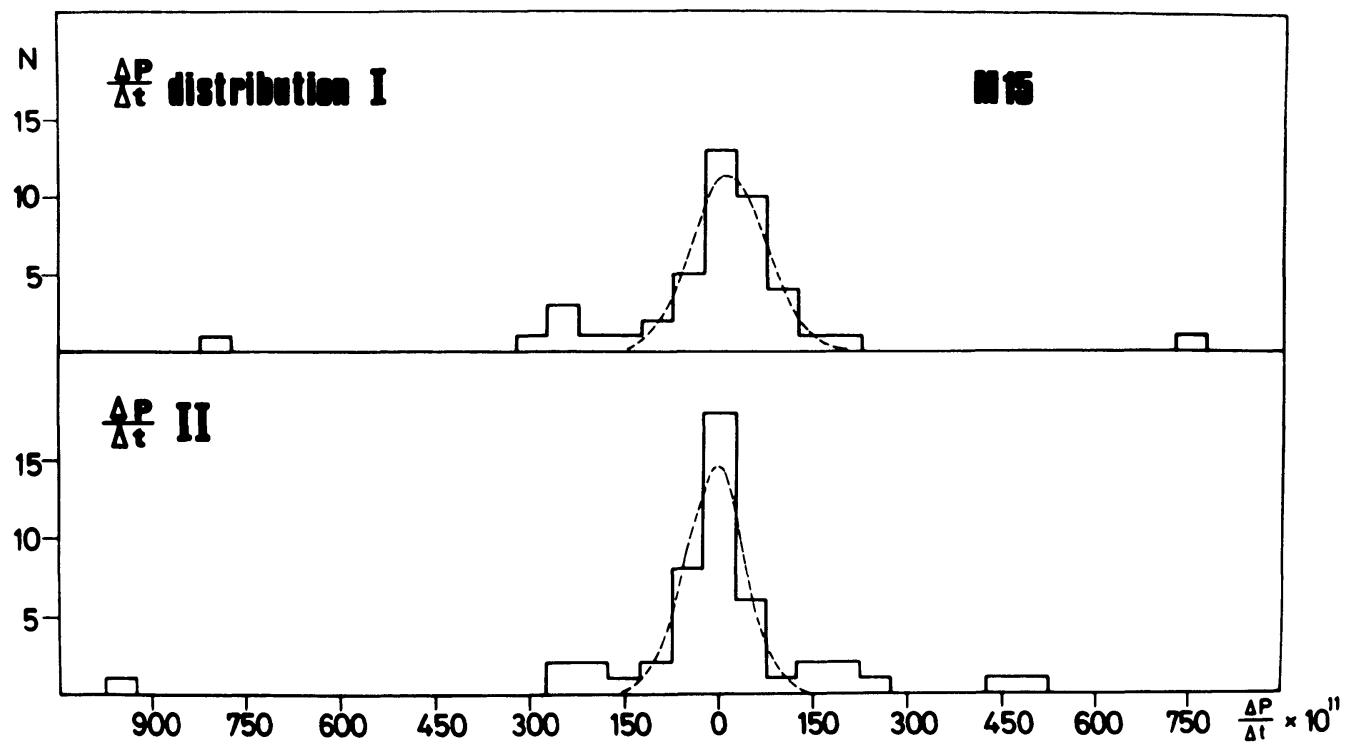

Fig. 2. For distribution $I \quad \sigma=60, x_{0}=+15$; for distribution II $\sigma=46, x_{0}=-7$.

the resulting O-C diagrams, even if these shapes are irregular or unusual. If the O-C residuals are only considered which do not contradict a parabola-like curve we might be deprived of evaluating the entire material available (Smith and Sandage, 1981). In the course of time further distributions can be made and the inclusion into the investigation of more clusters richer in variables will give more significant results.

Thanks are due to Drs. K. Olah, L. Pocs and B. Szeidl for helpful discussions and advice.

\section{REFERENCES}

Barlai, K.: 1984, Comm. Konkoly Obs., Budapest, No. 85 (in press) Belserene, E.P.: 1973, in Variable Stars in Globular Clusters and in

Related Systems, IAU Coll. 21, ed. J.D. Fernie, D. Reidel, Dordrecht, pp. 105-111

Smith, H.A. and Sandage, A.: 1981, Astron. J. 86, pp. 1870-1881

Sweigart, A.V. and Renzini, A.: 1979, Astron. Astrophys. 71, pp. 66-78 Szeidl, B.: 1975, in Variable Stars and Stellar Evolution, IAU Symp. 67 , eds. V.E. Sherwood, L. Plaut, D. Reidel, Dordrecht, pp. 545-552 


\section{DISCUSSION}

Cox: Did you look at any of the double mode variables?

Barlai: Yes, I did. From the 10 double mode RR Lyrae studied in your paper six have been measurable on our plates: V39, 26, 30, 31, 54 and 17. One of them, V26, makes a big jump from the left wing of distribution I to the right wing of distribution II. V39 with a distance of $\sim 40$ between its positions gets from the Gaussian part of distribution I to the right wing of distribution II. The remaining variables are included in the "noisy" part of the distributions. 